\title{
Editorials
}

\section{A new approach to patients with lower urinary tract symptoms}

Lower urinary tract symptoms (LUTS) are prevalent and bothersome symptoms, both for males and females. ${ }^{1}$ Most attention is given to LUTS in older males because in this group the prevalence is high and doctor visits are more common. ${ }^{2}$ The possibilities for physicians - both GPs as well as urologists - to encounter LUTS in daily practice are limited. The 2010 guideline The Management of Lower Urinary Tract Symptoms in Men, by the National Institute for Health and Clinical Excellence (NICE), presents an authority-based algorithm for this patient group. Education about the natural history of LUTS and the reassurance that LUTS are not caused by prostate cancer are important specific anchors for GPs and their patients. ${ }^{3.4}$ Treatment options for GPs are restricted to physiotherapy, alphablocking agents, 5-alpha reductase inhibitors, anticholinergics, or combination therapy. ${ }^{3}$ Urologists may offer operative treatment in those with severe symptoms not responding to non-invasive treatment.

The efficacy and effectiveness of treatments for LUTS in older males are limited. ${ }^{3}$ The probable explanation is that LUTS are multifactorial in their origin, whereas the therapeutic approach is simplistic, targeting specific organs.

\section{DETERMINING THE CAUSE}

Prostatic pathophysiology - especially prostate enlargement and prostate obstruction - was long thought to be the explanation for (nearly all) LUTS in older males and most of the drugs approved for LUTS in males aim to influence prostate function. Evidence is growing that this is a misconception. It is now clear that prostate enlargement or benign prostate obstruction is not the common cause of male LUTS. ${ }^{5}$ Many men with LUTS do not have prostate enlargement nor prostatic obstruction, whereas many men with an enlarged prostate do not have LUTS. It is also clear that solitary bladder dysfunction is hardly ever the predominant cause. ${ }^{5}$

It is not 'obstruction' or 'storage' that is the problem, but at present a 'Gordian knot', or unsolved problem, for which none of the current hypotheses is the cutting knife.

\section{PROBLEMS WITH GUIDANCE}

National and international guidelines, such as those on the treatment of patients with shoulder complaints or with low back pain, have shown a change from defined diseases and disorders into a more heterogeneous, non-specific, concept of symptoms. A similar change is needed with regard to LUTS. The NICE guideline states that:

In men, the most common cause is benign prostate enlargement ... which obstructs the bladder outlet.' 3,4

The NICE guideline was published long after a key-article on the explanation of LUTS in males and females. ${ }^{5}$ In that article, the results of an expert panel approach were presented and the conclusions were clear:

'It is misleading to attribute individual symptoms to sex differences or to a specific underlying organ. LUTS are a non-sexspecific, non-organ-specific group of symptoms, which are sometimes agerelated and progressive. 5

We do not understand why the authors of the NICE guideline did not explicitly follow that explanation, but instead hold on to the organ-targeted explanation. In this respect there is a difference between the complete guideline ${ }^{3}$ - recognising the multifactorial origin - and the guideline summary ${ }^{4}-$ presenting prostate enlargement as the predominant cause of LUTS in men. It seems that they have chosen to follow the majority of physicians and researchers (including the authors of this comment) who, in the past decades, seem to have worked as lemmings heading for only one direction: the prostate. An explanation might be that leaving the prostatehypothesis results in a vacuum, both in etiologic theory and the diagnostic and treatment practice: leaving an organoriented explanation ('diagnosis') results in a (fear for) lack of explicable treatment possibilities. That is, the evidence for other approaches is small. However, holding on to the prostate hypothesis will keep our focus in the wrong direction.

\section{FUTURE RESEARCH}

To help men (and women) with LUTS appropriately in the future, it is necessary to change our approach and look more precisely at the multifactorial origin of LUTS. For this, new studies are needed, both observational epidemiological studies as well as studies testing diagnostic and treatment algorithms (such as presented in the NICE guideline). Those studies should focus on the more real life heterogeneous case-mix of patients with LUTS, rather than use strict inclusion criteria. Strict inclusion criteria have lead (and will lead) to results for unrecognisable small subgroups of patients leaving most patients out in the cold. Their use may lead to hypotheses that are not applicable to the majority of patients with LUTS.

\section{RECOMMENDATIONS FOR CURRENT TREATMENT}

The pharmacological approach of this patient group should also be changed into a more patient-centred approach recognising the multifactorial origin of symptoms. We believe that this is more important than changing reimbursement strategies, such as the Quality and Outcomes Framework, to increase the incentives to improve care, as was suggested in a review of the NICE guidelines. ${ }^{6}$ Increasing knowledge with well-conducted research may support GPs and urologists in providing the care that patients with LUTS need. Using a proper vocabulary to define the problem of these patients also remains important.?

In the meantime, we have to acknowledge the limitations in daily practice. This means that we should explicitly discuss the uncertainties and lack of scientific evidence with patients. Some

\section{"National and international guidelines ... have shown a change from defined diseases and disorders into a more heterogeneous, non-specific, concept of symptoms. A similar change is needed with regard to LUTS.}




\section{"In the meantime, we have to acknowledge the limitations in daily practice. This means that we should explicitly discuss the uncertainties and lack of scientific evidence with patients.}

GPs may already have done this according to their knowledge about the shift of ideas about the aetiology and the consequences for daily practice. But the quoted remarks from the NICE guideline do not help them. We must explain that what we can do is follow an (educated) trial-and-error approach, based on what we do know.

We suggest the following scheme for daily (general) practice. Men who consult for LUTS need to be taken seriously by taking a full history, including possible thoughts and fears surrounding the symptoms. This is very important as there are often many underlying questions which may be the true reason for encounter. Answering these underlying questions may often be the key to a successful consultation, even if the symptoms are not altered by active treatment.

Physical examination should include a digital rectal examination (DRE), especially when patients fear prostate cancer. Although the objective value is limited, DRE will often meet the expectations of the patient. If no clear abnormalities in both the history and physical examination are found, patients can be reassured that prostate cancer is not the cause of their symptoms, but one cannot state categorically that such patients do not have prostate cancer. Performing a prostate-specific antigen test in these patients equates to unselected prostate cancer screening. The efficacy of such screening is not established, and it may possibly be harmful. ${ }^{8}$

We suggest as a next important step in the consultation to collect frequency volume charts for additional data on voiding function. ${ }^{9}$ GPs can explain the multifactorial cause of LUTS in males and females. Additionally, normal values - especially for voiding frequency - can be shown.

If treatment is preferred, GPs should explain that this requires a trial and error approach, as in most patients it is unclear which causal factor should be targeted. In accordance to the NICE guideline, patients who wish to receive active treatment are likely to receive alpha blocking agents. In males with predominantly irritative symptoms, antimuscarinic agents can be added, although the clinical effect of such drugs is limited. ${ }^{3,4}$ Only in those men with truly enlarged prostates, for whom surgical treatment is not an option leither due to patient preference or comorbidity), 5-alpha reductase inhibitors can be advised. It should be explained that this is a long-term treatment and the aim of such combination treatment is to decrease the change of symptom aggravation, not symptom reduction. ${ }^{9}$ For those patients who do not respond to drug treatment, specialised care by a urologist may be the final step, but also here, surgical treatment only focuses on the prostatic obstruction, and therefore pros and cons should be weighed carefully.

\section{Marco H Blanker}

GP and Epidemiologist, University of Groningen, University Medical Centre Groningen, Department of General Practice, Groningen, the Netherlands.

\section{Roelf JC Norg,}

GP, Maastricht University Medical Centre, Department of General Practice \& Department of Internal Medicine, Caphri School for Public Health and Primary Care, Maastricht, the Netherlands.

GP, University of Groningen, University Medical Centre Groningen, Department of General Practice, Groningen, the Netherlands.

\section{Competing interests}

All authors are members of the Male LUTS College of General Practitioners, but declare no other competing interests.

\section{Acknowledgements}

The authors thank the other members of the Male LUTS working committee of the Dutch College of General Practitioners for their input in the discussion. The members are: Simone A Breed, GP; Rien Klomp GP; Anja de Vries, GP; René J Wolters, GP.

\section{DOI: 10.3399/bjgp12X652184}

\section{Wouter $\mathrm{K}$ van der Heide,} guideline revision working group of the Dutch

\section{ADDRESS FOR CORRESPONDENCE}

\section{Marco Blanker}

University of Groningen, University Medical Centre Groningen, Department of General Practice,

Antonius Deusinglaan 1, P.O. Box 196, 9700 AD,

Groningen, the Netherlands.

E-mail: blankerabelvederelaan.nl

\section{REFERENCES}

1. Kupelian V, Wei JT, O'Leary MP, et al. Prevalence of lower urinary tract symptoms and effect on quality of life in a racially and ethnically diverse random sample: the Boston Area Community Health (BACH) Survey. Arch Intern Med 2006; 166(21): 2381-2387.

2. Wolters $R$, Wensing $M$, van Weel $C$, et al. Lower urinary tract symptoms: social influence is more important than symptoms in seeking medical care. BJU Int 2002; 90(7): 655-661.

3. National Clinical Guideline Centre. The management of lower urinary tract symptoms in men. London: National Clinical Guideline Centre at The Royal College of Physicians, 2010.

http://wnw.nice.org.uk/nicemedia/live/12984/ 48554/48554.pdf (accessed 12 Jun 2012).

4. National Institute of Clinical Excellence. Lower urinary tract symptoms. The management of lower urinary tract symptoms in men. NICE clinical guideline 97. London: NICE, 2010.

www.nice.org.uk/nicemedia/live/12984/48557/ 48557.pdf (accessed 12 Jun 2012).

5. Chapple CR, Wein AJ, Abrams P, et al. Lower urinary tract symptoms revisited: a broader clinical perspective. Eur Urol 2008; 54(3): 563-569.

6. Jones C, Hill J, Chapple C; Guideline Development Group. Management of lower urinary tract symptoms in men: summary of NICE guidance. BMJ 2010; 340: c2354.

7. Abrams P. The term $\mathrm{BPH}$ is misused. BMJ 2008; 336(7641): 405

8. Ilic D, O'Connor D, Green S, Wilt TJ. Screening for prostate cancer: an updated Cochrane systematic review. BJU Int 2011; 107(6): 882-891.

9. Sooriakumaran P, Brown C, Emberton M Frequency volume charts should be used in men with lower urinary tract symptoms. Int $J$ Surg 2005; 3(2): 147-150.

10. McConnell JD, Roehrborn CG, Bautista OM, et al. The long-term effect of doxazosin, finasteride, and combination therapy on the clinical progression of benign prostatic hyperplasia. N Engl J Med 2003; 349(25): 2387-2398 